\title{
Application of behavioral therapies in adult and adolescent patients with chronic migraine
}

\author{
Randall E. Weeks
}

(C) Springer-Verlag Italia 2013

\begin{abstract}
Patients with chronic migraine (CM) headaches present some of the most difficult treatment challenges for headache practitioners. Attention to psychological and behavioral issues become significant treatment considerations as the frequency of a patient's headaches increases, there is increased disability secondary to headaches, and/or there is inadequate response to usually effective treatment. Recent research has identified a variety of risk factors (including medication overuse) that appear to be associated with the escalation of the frequency and severity of migraine headache and are amenable to behavioral (nonpharmacological) treatment. The present article will highlight therapies that may be effective in the treatment of patients with CM headache.
\end{abstract}

Keywords Chronic migraine headaches - Cognitive/ behavioral treatment for headaches - Medication overuse headache

\section{Introduction}

The clinical presentation of migraine headaches is highly prevalent $[1,2]$ and affects not only individual patients (and their families) [3], but also, social and economic systems due to the direct and indirect costs of disability due to headaches $[4,5]$. Population-based studies have shown that most patients with migraine (1) fail to seek medical care for their headaches, (2) nearly half never receive a headache diagnosis, and (3) their headaches are

R. E. Weeks $(\square)$

The New England Center for Headache,

30 Buxton Farm Road, Stamford, CT 06905, USA

e-mail: rewphd044@aol.com sub-optimally treated [6]. Such findings are especially problematic as migraine headache may represent a progressive disorder in that episodic migraine (EM) sufferers develop transformed migraine to the chronic form at the rate of $2.5 \%$ per year [7].

Results of studies of global prevalence of chronic migraine (CM) have suffered from the application of different criteria and definitions of the disorder across studies. They are complicated further by heterogeneous interpretations of what constitutes medication overuse headache (MOH) and how it relates to CM. A previous review [8] estimated the global prevalence of CM appeared to be well under $1 \%$ of the adult population. Consistent with these data, recently published results from the American Migraine Prevalence and Prevention Study (AMPP) [9] found that the prevalence of $\mathrm{CM}$ in the population of the United States (US) was approximately $1 \%$. The prevalence of CM in adolescents in a US population-based study has been found to be $0.79 \%$ [10].

Using less restrictive criteria (CM defined as the occurrence of migraine headaches on 15 or more days per month for at least 3 months [11] and including patients with $\mathrm{MOH}$ ), the prevalence was found to range between 1 and $5 \%$ in adults [8] and $1.75 \%$ in adolescents [10]. It has been noted that even at the lower end of these estimates, the prevalence of $\mathrm{CM}$ was in the same range as epilepsy [12].

Previous research has identified clusters of patient variables in patients with $\mathrm{CM}$. In the AMPP study [9], CM was found to be highest among females, in mid-life, and in households with the lowest annual income. Relative to EM patients, individuals with $\mathrm{CM}$ had greater headache-related disability [13, 14], lower socio-economic status [15], poorer health-related quality of life [16], and higher direct and indirect costs [17]. Other studies have noted that CM 
populations have been found to have higher rates of a variety of co-morbid conditions (psychiatric disorders, pain states, respiratory illness, and disease associated with cardiac risk) [18]; greater polypharmacy and social impediments [19]; and post-traumatic stress disorder [20]. Such findings highlight the complexities and co-morbidities in such patients, which necessitate a comprehensive treatment strategy involving both pharmacological and behavioral strategies.

A variety of "modifiable" risk factors have been identified regarding headache progression in migraine patients [21]. These include increase in attack frequency, obesity, medication overuse, stressful life events, caffeine overuse, and snoring/sleep apnea. Such variables offer pharmacological and non-pharmacological treatment opportunities for the headache clinician.

As the frequency of migraine headache increases, patients will often increase consumption of acute headache medications, which paradoxically causes "rebound headaches" and leads to chronic MOH [22, 23]. This is a critical variable to consider in the treatment of patients who present with $\mathrm{CM}$.

\section{Behavioral assessment}

Patients with CM present with a history of experiences and expectations with respect to pharmacological and behavioral treatments. There may be some pessimism regarding any new treatment plan as the course of CM is usually an increase in the frequency, severity, and disability of headache as previous treatments have failed to be effective. Given the comorbid complexities that seem to exist in $\mathrm{CM}$ patients, effective diagnosis and management of headache may only occur after an assessment of the specific needs of the individual patient, the context in which the patient's head pain occurs, and the impact on the individual's life. The framework for treatment begins with the initial behavioral assessment.

Assessment of compliance with previous pharmacological and non-pharmacological interventions needs to be assessed as it has been shown to be an important treatment issue in headache and other medical patients [24, 25]. There is a vast literature that concludes that many of these patients fail to take abortive medications [26, 27] and preventive medications [28, 29] as prescribed. Treatment drop-out and inconsistency in appointment keeping may be problems for the treating clinician. Given the comorbid complexities of CM patients noted above as well as the likelihood that the headaches have progressed due to ineffective treatment, adherence should be viewed as an ongoing treatment variable and should be stated as such at the initial evaluation.
As noted above, recent research has identified "modifiable" risk factors for migraine and headache progression [21]. Assessment of these variables (attack frequency, obesity, medication overuse, stressful life events, caffeine overuse, and snoring/sleep apnea) are important data regarding the formulation of a comprehensive treatment strategy. Behavioral treatment approaches (especially when implemented early in the patient's headache history) are indicated to address these risk factors. Patient education is important.

Medication overuse headache occurs when usually effective abortive agents become "overused", and their consumption leads to decreased efficacy regarding headache relief. This could lead to a "rebound" phenomenon in which the use of abortive agents paradoxically increases the frequency and severity of head pain. Numerous studies have noted the need to eliminate these agents from the patient's treatment as ongoing "overuse" negatively impacts both pharmacological and non-pharmacological interventions [30, 31].

When evaluating patients and formulating a treatment strategy, a careful medication history is important. It is important to assess not only what medications the patient has taken (both prescription and over-the-counter preparations), but also, at what point in the pain process that the patient medicates. Many patients medicate in the anticipation of pain escalation, which leads to increased (and possibly unnecessary) medication consumption. Other patients fail to take their migraine medication early in the headache process [32], which minimizes the likelihood of becoming pain-free, increases the duration of the headache, and ultimately, leads to the use of more abortive medication. A recent study involving migraine patients [33] found that $45 \%$ of migraine patients delayed treatment beyond what was advised. Reasons listed included "the need to reserve medications for a more severe headache" (51.2\%), "did not have medication on hand" (34.9\%), "health plan limits" (27\%), "inability to swallow because of nausea" (18.7\%), "not having access to fluid" (17.3\%), and "not having privacy" (13.5\%).

The use of pain medication has long been frowned upon for the treatment of migraine headache. A recent, population-based study [34] found that the use of barbiturates or opiates led to an increased risk of developing transformed migraine. Such data were consistent with earlier clinicbased studies in which migraine patients treated with opioids for other biological or pain issues were likely to develop transformed migraine [35-37]. Hence, extreme caution should be the rule in the use of opioids or butalbital agents in migraine patients (even for other medical issues).

Identification of subgroups of patients with $\mathrm{MOH}$ has been shown to predict treatment outcome. One approach [38] divides $\mathrm{MOH}$ patients into two groups-those with 
Type 1 or "simple" histories (relatively short-term drug overuse, relatively modest amounts of overused medications, minimal psychiatric contribution, and no history of relapse of after drug withdrawal) vs. those with Type 2 "complex" histories (multiple psychiatric co-morbidities and history of relapse). It is believed that the Type 1 group should be treated aggressively to prevent evolution into Type 2 patients. Behavioral treatments are strongly recommended in addition to appropriate pharmacotherapy. It is essential that the Type 2 group have both pharmacological and non-pharmacological treatments.

In sum, obtaining a complete medical, medication, and behavioral/psychosocial history is critical. The patient's subjective sense of what medication has been the most effective in the past may also be revealing.

Habit history regarding the patient's current and previous nicotine, caffeine, alcohol, and substance use should be assessed. Sleep difficulties or disturbance should be evaluated. Consistency in eating habits, caffeine consumption, and sleep patterns should be assessed as all can contribute to headache and may be markers of underlying affective disturbance. Previous psychiatric history and symptoms should be noted.

With respect to the systems in the patient's life, there should be an assessment of the family's reaction to the patient's headaches as well as any areas of family conflict. This is especially critical in the evaluation of a child with headache. The impact of the patient's headaches on academic/vocational systems should be assessed. The number of absences and degree of disability should be noted. Variables such as job security in adults or the need for "homebound" tutoring with adolescents should be explored. Evidence of secondary gain from headache disability should be assessed.

In sum, the information obtained at the clinical interview provides clinicians with the basic data to make a preliminary headache diagnosis. Symptom markers begin to emerge that could reflect risk factors for migraine escalation as well as depict comorbid psychiatric issues that may require pharmacological and behavioral treatment. Each patient is required to keep a headache calendar that is brought to each meeting with the clinician. The calendar documents the frequency, intensity, duration, and disability related to headache. The diary also records the type and amount of medication taken as well as relief efficacy. Menstrual days and other potential dietary and environmental triggers are noted. The headache calendar provides reliable, objective information for both the clinician and the patient, and it serves as a time-saving tool for the clinician to accurately judge clinical changes and treatment efficacy.

Education is a key component regarding not only the clinical diagnosis, but also, the proposed treatment options and treatment course. Both pharmacological and behavioral treatments should be considered. The treatment philosophy is based on the patient being an "active participant" in the treatment process. Patient questions and questions from significant others should be answered at this point.

\section{Behavioral treatment}

It should be emphasized that behavioral and other nonpharmacological treatments are not anti-pharmacological. The combination of both pharmacological and nonpharmacological treatment has been shown to be superior to each individually [39] and appear to maximize long-term therapeutic benefit [40]. In addition, effective non-pharmacological strategies help to ensure pharmacological treatment compliance which has been shown to be a significant problem with headache patients [24, 25].

The American Academy of Neurology-U.S. Consortium [41] noted the following reasons that cause migraine patients to seek behavioral and other non-pharmacological treatment for migraine headache:

1. Patient preference.

2. Poor tolerance/poor response to preventive medications.

3. Medical contraindications to medications.

4. Pregnancy, planned pregnancy, or nursing.

5. History of overuse of acute care medications.

6. Significant stress or deficient stress/pain coping strategies.

Goals of non-pharmacological treatment included:

1. Reduced frequency/severity of headache.

2. Reduced headache-related disability.

3. Reduced reliance on poorly tolerated or unwanted pharmacotherapy.

4. Enhanced personal control of pain.

5. Reduced headache-related distress and psychological symptoms.

Clinically, it has been determined that behavioral treatments are effective,

More than 100 empirical studies have examined the efficacy of bio-behavioral therapies in headache. The American Academy of Neurology-U.S. Consortium published evidence-based guidelines for migraine headache treatment and concluded that relaxation training, thermal biofeedback combined with relaxation training, EMG biofeedback, and cognitive-behavior therapy were effective treatment options for migraine [41]. Meta-analytic reviews have shown that such non-pharmacological treatments have been effective for both migraine and tension-type headaches [42]. 
Patients benefit from a detailed educational program that highlights the complex nature of headache difficulties as well as the factors that appear to be related to the escalation of the frequency and severity of migraine headache. What follows is a model of behavioral treatment for CM patients. Treatment strategies are outlined that address the modifiable risk factors for the escalation of migraine from episodic to chronic [21].

\section{Medication overuse}

An essential component to the treatment of $\mathrm{MOH}$ is the withdrawal of the "overused" medication [43]. Such strategies involve effective communication with the patient about the discontinuation/detox schedule regarding the offending agent [44]. Education regarding the "washout period" (the period of time that it may take the patient to experience significant improvement) is critical. Treatment typically takes place on an outpatient basis if patients have a relatively short period of medication overuse, use only one or two substances at low doses, have adequate support systems, and are highly motivated [38]. Patients with more complicated medication regimens and greater medical/ psychiatric co-morbidities may require inpatient treatment.

Headache calendars or diaries are necessary to document adequately the patient's treatment progress. Such documentation should include the detox schedule and the doses/frequency/limits of medication allowed. This could involve the use of a medication "bridge therapy" to help in the reduction. Appropriate preventive medications should be started. Patients may need to be seen frequently early in the treatment process of $\mathrm{MOH}$ in order to assist them during the "washout period".

In addition to the above medication strategies, behavioral treatments (see below) are also indicated. As noted previously, the combination of both pharmacological and non-pharmacological treatment has been shown to be superior to each individually [39] and appears to maximize long-term therapeutic benefit in $\mathrm{MOH}$ patients [40]. In addition, effective non-pharmacological strategies help to ensure pharmacological treatment compliance (which has been shown to be a significant problem with headache patients) [24, 25].

\section{Increased attack frequency}

The factors that act as headache triggers vary from person to person. Among the many triggers identified in the literature are dietary factors, fasting and skipping meals, changes in sleep patterns, the experience of acute and chronic stress, effects of overexertion, the impact of hormonal changes, susceptibility to weather changes, and reaction to sensory stimuli.
Patients are encouraged to investigate and validate which dietary and environmental agents may be potential headache triggers. Headache calendars facilitate this process by highlighting potential foods that have been identified in the migraine literature to allow the patient to record what, if any, impact such agents may have on the patient's headaches. Different foods (and caffeine and alcohol consumption) are noted. Although much has been written about dietary factors in relation to migraine, few research protocols have been well designed. Though widespread efficacy has not been established, these studies indicate that dietary modifications might be of some benefit for some patients.

Chronic migraine patients are encouraged to modify behavioral factors in an effort to decrease headache frequency and severity. They are encouraged to keep to normal sleep/wake patterns (even on weekends). Naps and "oversleeping" are to be avoided. Patients are also advised to eat nutritious meals at regular intervals.

Patients also learn behavioral strategies to help with headache control and begin setting behavioral goals such as improving time management, increasing aerobic exercise, and participating in more pleasurable activities. Patients who exhibit aspects of the Type-A behavior pattern are taught how to modify such behavior.

A preventive medication is often helpful to reduce headache attack frequency [45, 46]. The issue of medication adherence may be a treatment concern as approximately $25-50 \%$ of headache patients were found to have failed to take preventive medication correctly [28, 29]. A variety of factors have been shown to affect whether patients take preventive medications as prescribed. Positive factors include whether the clinician involves the patient in decision-making, whether the physician takes time to explain potential side-effects, and whether the physician relates to the patient published efficacy data [47]. Negative factors regarding adherence included whether the proposed preventive medication could cause weight gain or sedation. Other variables that could affect adherence include simplifying treatment protocols, having fewer doses each day, and explaining the length of time before treatment efficacy would be expected.

\section{Stressful life events}

Patients are taught self-regulation techniques and the importance of cognitive and behavioral coping skills. A major component of this type of intervention is to teach the patient coping skills designed to alleviate both the sensory and reactive components of the total pain experience. The sensory component involves the perception of physical sensations (including pain) that can be altered through relaxation therapies and biofeedback. The reactive 
component consists of thoughts and feelings that accompany headache and may lower the pain threshold, lead to "problematic behaviors" (e.g., overuse of abortive medications), heighten levels of sympathetic arousal, and possibly, increase neuronal hyperexcitability. The patient may display a decreased sense of mastery and control (increased helplessness). Cognitive-behavioral interventions help modify the reactive component by emphasizing skills that increase the patient's ability to cope with the pain and to reduce headache-related distress.

Initial treatment sessions are directed toward the sensory component by using relaxation training and biofeedback to teach physiological self-regulation. Relaxation therapies include several techniques that target the entire body and enable patients to develop greater body awareness, achieve an overall relaxed state, and gain confidence regarding physiological control.

Biofeedback training targets specific physical responses believed to contribute to increased headache susceptibility and maintenance of pain. The instrumentation "feeds back" immediate objective information about biological processes that is normally beyond patients' awareness and control. The feedback may be visual or auditory. Patients learn to bring biological processes under voluntary control, and thereby, lower arousal. Through training, the processes are "shaped" in a more adaptive direction (i.e., muscle tension decreased in pericranial muscles and finger temperatures increased) and become "overlearned" (i.e., take place automatically without the patient's conscious awareness). The biofeedback program is a step-by-step approach that builds each patient's skills over a series of sessions. Patients must master each skill before moving on to the next, and they should be encouraged to use audiocassettes for home practice.

The next part of the program consists of cognitive/ behavioral pain and stress management strategies that focus on the reactive component of the pain experience. Patients learn to identify and modify distress-related thoughts and maladaptive styles of thinking that can contribute to headache susceptibility. This type of therapy emphasizes the role of thoughts, perceptions, belief systems, self-evaluations and appraisals that influence emotional states, physiology, and behavior. Techniques are aimed at providing patients with a set of problem-solving and coping skills they can use in a wide range of situations that trigger and maintain headache. Distress-related thoughts and negative self-talk (e.g., "Why me?"... “I can't believe I'm getting another migraine"... "It's no use") mediate poor outcome through a variety of mechanisms including depressed mood, increased anxiety level, decreased pain threshold, poor treatment compliance, and overuse of pain medications.

Many patients magnify the negative aspects of their situations and become fatalistic and helpless. They often have low tolerance for pain and believe they are unable to control their head pain. They develop an external locus of control and start to look for a "magic pill". To develop alternative cognitive responses to the experience of recurrent and severe head pain, these patients are taught positive self-statements that redefine their headaches as manageable events that can be resolved. Patients rehearse adaptive cognitive and behavioral responses to the development of a migraine and develop task-relevant management skills to reduce the anxiety that often magnifies symptoms.

Self-statements help patients (1) prepare for an attack, (2) manage initial symptoms, (3) handle critical moments during the attack, and (4) act adaptively during the postheadache phase. Patients become keen observers, prepare to cope adaptively, and avoid becoming hypervigilant to pain sensations. Many headache patients exhibit irrational ideation about the possibility of the onset of headache and underestimate their coping skills. Additionally, these cognitive coping strategies foster adherence to drug regimens, reduce headache-related distress, and result in better management of acute migraine attacks.

There is an impressive literature that notes headache (especially migraine) is highly comorbid with depression, anxiety, and bipolar affective disorder [48]. Some patients require formal cognitive/behavioral psychotherapy to address these underlying mood disorders.

\section{Caffeine overuse}

Setting limits regarding caffeine consumption may be an important part of the treatment strategy. Some clinicians believe that total elimination of caffeine is necessary to maximize headache reduction. Many believe "modest" caffeine consumption is allowable (less than two cups per day). Patients need to be reminded that this is "cups" not "mugs" and that the caffeine should be consumed each day (i.e., do not skip days of consuming caffeine). Patients should also be reminded that many medications may contain caffeine.

\section{Snoring/sleep apnea}

The importance of quality, consistent sleep has been found to be a variable that separates episodic vs. CM patients [49]. It has also been implicated as a cause of increased frequency of headaches in adolescent patients [50]. Appropriate referral/ workup should be ordered if patient and/or significant other report excessive snoring, cessation of breathing, etc.

\section{Obesity}

Increased physical activity, dietary restrictions, etc. may be necessary to reduce likelihood of "inflammatory soup" 
[51] and an increase in headache frequency, severity, and refractoriness to treatment. Screening and behavioral management for issues with weight should be part of the treatment process.

\section{Summary}

Clinicians who treat headache patients need to be aware of bio-behavioral issues in both the assessment and treatment of such patients. Effective treatment begins with a thorough diagnostic interview and the introduction of a headache diary as a tool for self-monitoring. Educating patients about headache mechanisms and treatment allows a collaborative relationship between patient and clinician. Training healthy physiological responses and developing behavioral and cognitive strategies to manage pain, empowers patients regarding pain management and reduces helplessness.

Chronic migraine complicated by $\mathrm{MOH}$ is a treatment challenge for the clinician. With the identification of $\mathrm{MOH}$ and other "risk factors" in headache progression, comprehensive treatment early in the patient's headache history is indicated using both pharmacological and behavioral treatment strategies. Effective treatment begins with establishing a therapeutic relationship with patients in which the clinician underscores the expectation of treatment compliance on the patient's part. Treatment goals must be defined behaviorally and objectively. Patients must be "active participants" in the management of their headache disorder.

Conflict of interest The author certify that there is no actual or potential conflict of interest in relation to this article.

\section{References}

1. Stewart WF, Lipton RB, Celentano DD et al (1992) Prevalence of migraine headache in the United States: relation to age, income, race, and other socio-demographic factors. JAMA 267:64-69

2. Lipton RB, Hamelsky SW, Stewart WF (2001) Epidemiology and impact of headache. In: Silberstein SD, Lipton RB, Dalessio DJ (eds) Wolff's Headaches and other Head Pain, 7th edn. Oxford University Press, Oxford, pp 85-107

3. Lipton RB, Hamelsky SW, Kolodner KB et al (2000) Migraine, quality of life, and depression: a population-based case-controlled study. Neurology 55:629-635

4. VonKorff M, Stewart WF, Simon DJ et al (1998) Migraine and reduced work performance: a population-based diary study. Neurology 50:1741-1745

5. Hu HX, Markson LE, Lipton RB et al (1999) Burden of migraine in the United States: disability and economic costs. Arch Intern Med 159:813-818

6. Lipton RB, Bigal ME (2007) Ten lessons in the epidemiology of migraine. Headache 47(Suppl 1):S2-S9

7. Bigal ME, Serrano D, Scher A et al (2008) Acute migraine medications and evolution from episodic to chronic migraine: a longitudinal population-based study. Headache 48(Suppl 4):S3
8. Natoli JL, Manack A, Dean B et al (2009) Global prevalence of chronic migraine: a systematic review. Cephalalgia 30:599-609

9. Buse DC, Manack AN, Fanning KM et al (2012) Chronic migraine prevalence, disability, and socio-demographic factors: results from the American migraine prevalence and prevention study. Headache 52:1456-1470

10. Lipton RB, Manack A, Ricci JA et al (2011) Prevalence and burden of chronic migraine in adolescents: results of the chronic daily headache in adolescents study (C-das). Headache 51: 693-706

11. Olesen J, Bousser MG, Diener HC et al (2006) New appendix criteria open for a broader concept of chronic migraine. Cephalalgia 26:742-746

12. Hauser WA, Annegers JF, Rocca WA (1996) Descriptive epidemiology of epilepsy: contributions of population-based studies from Rochester, Minnesota. Mayo Clin Proc 71:576-586

13. Bigal ME, Serrano D, Reed M et al (2008) Chronic migraine in the population: burden, diagnosis, and satisfaction with treatment. Neurology 71:559-566

14. Buse D, Manack A, Serrano D et al (2012) Headache impact of chronic and episodic migraine: results from the American migraine prevalence and prevention study. Headache 52:3-17

15. Buse DC, Manack A, Serrano D et al (2010) Socio-demographic and comorbidity profiles of chronic migraine and episodic migraine sufferers. J Neurol Neurosurg Psychiatry 81:428-432

16. Meletiche DM, Lofland JH, Young WB (2001) Quality of life differences between patients with episodic and transformed migraine. Headache 41:573-578

17. Stewart WF, Wood GC, Manack A et al (2010) Employment and work impact of chronic migraine and episodic migraine. J Occup Environ Med 52:8-14

18. Lipton RB, Buse D, Serrano D et al (2008) Differences in rates of common comorbid medical and psychiatric conditions in chronic and episodic migraine individuals. Headache 48(Suppl 4):S4

19. Ferrari A, Leone S, Vergoni AV et al (2007) Similarities and differences between chronic migraine and episodic migraine. Headache 47:65-72

20. Peterlin BL, Tietjen G, Meng S et al (2008) Post-traumatic stress disorder in episodic and chronic migraine. Headache 48:517-522

21. Bigal ME, Lipton RB (2006) Modifiable risk factors for migraine progression. Headache 46:1334-1343

22. Cupini LM, Calabresi P (2005) Medication-overuse headache: pathophysiological insights. J Headache Pain 6:199-202

23. Diener HC, Limroth V (2004) Medication-overuse headache: a worldwide problem. Lancet 3:475-483

24. Rains JC, Lipchik GL, Penzien DB (2006) Behavioral facilitation of medical treatment for headache--part I: review of headache treatment compliance. Headache 46:1387-1394

25. Rains JC, Penzien DB, Lipchik GL (2006) Behavioral facilitation of medical treatment for headache--part II: theoretical models and behavioral strategies for improving adherence. Headache 46:1395-1403

26. Spierings EL, Miree LF (1993) Non-compliance with follow-up and improvement after treatrment at a headache center. Headache 33:205-209

27. Holroyd KA, Cordingley GE, Pingel JD et al (1989) Enhancing the effectiveness of abortive therapy: a controlled evaluation of self-management training. Headache 29:148-153

28. Gallagher RM, Kunkel R (2003) Migraine medication attributes important for patient compliance: concerns about side effects could delay treatment. Headache 43:36-43

29. Mulleners WM, Whitmarsh TE, Steiner TJ (1998) Non-compliance may render migraine prophylaxis useless, but once-daily regimens are better. Cephalalgia 18:52-56

30. Mathew NT, Kurman R, Perez F (1990) Drug-induced refractory headache-clinical features and management. Headache 30:634-638 
31. Ward TN (2008) Drug-induced refractory headache. Headache 48:728-729

32. Golden W, Katic BJ, Hu H (2008) Factors associated with early treatment of acute migraine headache pain. Headache 48(Suppl): S42-S43

33. Golden W, Katic BJ, Hu H (2008) Why did migraine patients not treat migraine attacks as early as they desired? Headache 48(Suppl):S42

34. Bigal ME, Serrano D, Buse D et al (2008) Acute migraine medications and evolution from episodic to chronic migraine: a longitudinal population-based study. Headache 48:1157-1168

35. Wilkinson SM, Becker WJ, Heine JA (2001) Opiate use to control bowel motility may induce chronic daily headache in patients with migraine. Headache 41:303-309

36. Bahra A, Walsh M, Menon S et al (2003) Does chronic daily headache arise de novo in association with regular use of analgesics? Headache 43:179-190

37. Paemeleire K, Bahra A, Evers S et al (2006) Medication-overuse headache in patients with cluster headache. Neurology 67:109-113

38. Lake AE (2006) Medication overuse headache: bio-behavioral issues and solutions. Headache 46(Suppl.3):S88-S97

39. Holroyd KA, O'Donnell FJ, Stensland M, Lipchik GL (2001) Management of chronic tension-type headache with tricyclic antidepressant medication, stress-management therapy, and their combination: a randomized controlled trial. JAMA 285:22082215

40. Grazzi L, Andrasik F, D'Amico D et al (2002) Behavioral and pharmacologic treatment of transformed migraine with analgesic overuse: outcome at 3 years. Headache 42:483-490
41. Campbell JK, Penzien DB, Wall EM (2000) Evidence-based guidelines for migraine headaches: behavioral and physical treatments. Available at: http://www.aan.com/professionals/practice/ pdfs/g10089.pdf

42. Andrasik F (2007) What does the evidence show? Efficacy of behavioural treatments for recurrent headaches in adults. Neurol Sci 28:S70-S77

43. Mathew NT (1990) Drug-induced headache. Neurol Clin 8: 903-912

44. Rapoport A (2008) Medication overuse headache: awareness, detection, and treatment. CNS Drugs 22:995-1004

45. Lipton RB, Bigal ME, Diamond M et al (2007) Migraine prevalence, disease burden, and the need for preventive therapy. Neurology 68:343-349

46. Packard RC, Brown F (1986) Multiple headaches in a case of multiple personality disorder. Headache 26:99-102

47. Rozen TD (2008) Migraine prevention: what patients want from medication and their physician. Headache 48:750-753

48. Hamelsky SW, Lipton RB (2006) Psychiatric comorbidity of migraine. Headache 46:1327-1333

49. Calhoun AC, Ford S (2007) Behavioral sleep modification may revert transformed migraine to episodic migraine. Headache 47:1178-1183

50. Gilman DK, Palermo TM, Kabbouche MA et al (2007) Primary headache and sleep disturbance in adolescents. Headache 47: 1189-1194

51. Nicholson R, Bigal M (2008) Screening and behavioral management: obesity and weight management. Headache 48:51-57 